\title{
Positive predictive value of ICD-10 codes to detect anaphylaxis due to vaccination: a validation study
}

Yonatan Moges Mesfin ${ }^{1}$, Allen C Cheng ${ }^{2,3}$, Aimy HL Tran ${ }^{4,5}$, Jim Buttery ${ }^{1,2}$

\section{Author affiliations:}

\author{
${ }^{1}$ Monash Centre for Health Research and Implementation, School of Public \\ Health and Preventive Medicine, Monash University, Melbourne, Victoria, \\ Australia \\ ${ }^{2}$ Monash Children Hospital, Monash Health, Melbourne, Victoria, Australia \\ ${ }^{3}$ Infection Prevention and Healthcare Epidemiology Unit, Alfred Health \\ Melbourne, Victoria, Australia \\ ${ }^{4}$ Department of Paediatrics, Monash University, Melbourne, Victoria, Australia \\ ${ }^{5}$ The Ritchie Centre, Hudson Institute of Medical Research, Melbourne, Victoria, \\ Australia
}

Correspondence: Yonatan Moges Mesfin

Monash Centre for Health Research and Implementation Locked Bag 29

Clayton VIC 3168

Australia

Email: Yonatan.Mesfin@monash.edu

Tel: $\quad+61401047325$

Total word count

$$
\text { Abstract }=236
$$

This is the author manuscript accepted for publication and has undergone full peer review but has not been through the copyediting, typesetting, pagination and proofreading process, which may lead to differences between this version and the Version of Record. Please cite this article as doi: $10.1002 /$ pds.4877

This article is protected by copyright. All rights reserved. 
Main document $=2494$

\begin{abstract}
Purpose: To validate the use of selected International Classification of Disease Codes $10^{\text {th }}$ revision (ICD-10) to predict (positive predictive value) anaphylaxis due to vaccination using emergency department (ED) data.
\end{abstract}

Methods: We conducted a retrospective study using ED encounter data from a large tertiary-care teaching hospital, Monash Medical Centre, Melbourne, Australia. We searched all ED encounters potentially due to anaphylaxis after vaccination, between 1 January 2010 and 31 December 2018, using ICD-10-CM codes T80.5, T80.6, T88.1, T88.6 and T78.2. Health records of potential cases were examined to determine if they met the Brighton Collaboration (BC) criteria for anaphylaxis. We calculated the PPV to evaluate the accuracy of the selected ICD-10-CM codes in predicting anaphylaxis due to vaccination.

Results: Of the 69 health records identified and reviewed, 29 (42.2\%) met the criteria for anaphylaxis regardless of the cause, and $24.6 \%$ (17/69) of records were confirmed as anaphylaxis triggered by vaccination (low positive predictive value). However, of the 23 records identified using ICD-10-CM code T80.5, 22 were classified as anaphylaxis

This article is protected by copyright. All rights reserved. 
cases regardless of the cause and 12 were anaphylaxis due to vaccination cases giving PPV of $95.7 \%$ and $52.2 \%$ respectively.

Conclusions: Given that there is no specific ICD-10-CM code for anaphylaxis due to vaccination, ICD-10-CM code T80.5 may be suitable to monitor anaphylaxis due to vaccination in the ED setting. The current study was conducted at a single centre, and needs to be confirmed by future multicentre studies.

Keywords: Anaphylaxis, International Classification of Disease Codes, Vaccination, Validation

\section{Key Points}

- Vaccine safety studies most often use diagnostic codes to identify potential adverse events following immunization from electronic health records.

- Anaphylaxis is a potentially life-threatening severe allergic reaction, and rarely occurs following immunization.

- $\quad$ ICD-10 code T80.5 achieved an average positive predictive value to identify anaphylaxis following immunization (AFI).

- Although coding is not an accurate indication of diagnoses; T80.5 can be used for tracking AFI in the emergency department setting.

This article is protected by copyright. All rights reserved. 


\section{Introduction}

Common vaccine adverse events are typically detected during pre-licensure clinical trials prior to approval for general public use. ${ }^{1}$ However, rare adverse events may either go undetected during clinical trials due to insufficient sample size or because the adverse events only occur in sub-populations that were not included in the trials. Adverse events following immunisation (AEFI) may cause health burdens on the vaccinated individuals and also negatively influence the public confidence in vaccination, leading to vaccine hesitancy. ${ }^{2}$ Thus, safety monitoring for vaccines continues after licensure to identify rare and unexpected AEFIs and changes in frequency of known ones.

In most countries, including Australia, passive surveillance of AEFI is considered the principal approach for monitoring the safety of vaccines after licensure, whereby healthcare providers, patients/caregivers and any concerned body voluntarily report AEFI. ${ }^{3,4}$ Passive AEFI surveillance, however, has well-known limitations including under-reporting and data incompleteness. ${ }^{3}$ Conversely, active surveillance of AEFI using routinely collected health care data has offered an opportunity to track vaccines safety in near real-time. ${ }^{5}$ Active AEFI surveillance studies often use linked data; exposure information (vaccination) from vaccination registry are linked with prespecified outcomes of interest identified from electronic records using computerised International Classification of Diseases (ICD) diagnosis codes. ${ }^{6}$ Moreover, studies also 
suggest that vaccine safety signals can be tracked using proxy measures of AEFI occurrences, such as post-vaccination healthcare utilisation/medical attendance rate. ${ }^{7}$ 8

The ICD-10-CM (10th revision, clinical modification) contains diagnostic codes assigned for AEFI related diagnoses, such as T80.5 (anaphylaxis due to serum/vaccine). However, anaphylaxis due to other serum products is also coded under T80.5. Other AEFI-related ICD-10-CM codes include T80.6 (other serum/vaccine reaction, not anaphylaxis), T88.1 (other complication following immunisation not elsewhere classified) and T88.0 (infection following immunisation). ${ }^{9}$ Anaphylaxis is a potentially life-threatening severe allergic reaction and very rare event. All vaccines have the potential to trigger anaphylaxis, with risk estimates ranging from 1-10 per 1 million dose distributed, depending on the vaccine studied. ${ }^{10-12}$ Vaccine safety studies most often use an ICD code to identify anaphylaxis from electronic healthcare record databases. However, there is scarcity of studies on the accuracy of AEFI-related ICD-CM codes, particularly on anaphylaxis due to vaccination. One study evaluated the accuracy of ICD-9 codes of anaphylaxis associated with medication and biologics of interest in general, and reported positive predictive values (PPV) ranging from $45.8 \%$ to $69 \% .{ }^{13}$ This study aimed to evaluate the PPV of selected ICD-10-CM codes to identify anaphylaxis following vaccination using an emergency department (ED) dataset, for which codes are potentially available in real-time. 


\section{Methods}

\subsection{Data source and study population}

We conducted a retrospective study using ED records at Monash Health, incorporating three emergency department sites in South-Eastern Melbourne, Australia. Monash Health is the largest health network in Melbourne, Australia, handling more than 206,000 emergency presentations each year. Monash Health includes Monash Children's Hospital, one of the largest children's hospitals in Australia with more than 65,000 annual ED presentations. ${ }^{14}$ All ED encounters with pre-selected ICD-10-CM codes between 1 July 2010 and 30 June 2018 were searched to identify possible hospital visits for anaphylaxis due to vaccination. We primarily considered ICD-10-CM code T80.5 to identify anaphylactic reactions due to serum, which includes vaccines. To capture other possible cases of anaphylaxis due to vaccination, we additionally selected two nonspecific codes: T80.6 (other serum reaction, not anaphylaxis) and T88.1 (other complication following immunisation not elsewhere classified). Moreover, we surveyed a random sample of health records coded with T88.6 (anaphylactic reaction or shock due to adverse effect of correct medicinal substance properly administered) and T78.2 (anaphylactic reaction/allergic reaction unspecified) to check for incorrectly diagnosed or coded anaphylaxis due to serum products including vaccines. We included all health records coded with T80.5, T80.6 or T88.1 for chart review.

This article is protected by copyright. All rights reserved. 


\subsection{Record validation}

Two reviewers independently reviewed the selected medical records and extracted the following information: patient age, gender, ED visiting date, chief presenting complaints, history of vaccination, exposure other than vaccination, vaccination date and time, type of vaccine administered, signs and symptoms, and onset (suddenness) of the symptom and signs. Collated information were then categorised into the three levels of anaphylaxis diagnostic certainty based on the Brighton Collaboration (BC) case definition (Table 1 and Table 2). ${ }^{11}$ For records where the two reviewers disagreed, medical records were re-checked and a final determination was made through discussion.

\subsection{Statistical analysis}

Our study outcome was the PPV of an anaphylaxis due to vaccination diagnosis, defined as the proportion of ED encounters with diagnosis code of T80.5, T80.6 or T88.1 who had level 1, 2, or 3 anaphylaxis based on BC case definition. We summarised data using descriptive statistics and determined the PPV with $95 \%$ confidence intervals (Cls). The PPV was calculated for individual ICD-10-CM codes and combinations of codes. In addition, PPVs were estimated according to age group, gender, calendar period and principal presenting problem. We calculated the Cohen's kappa score, which measured the level of agreement between reviewers in categorising records

This article is protected by copyright. All rights reserved. 
into $\mathrm{BC}$ case definition levels of diagnostic certainty. Analyses were conducted using STATA $/$ IC $^{\circledR}$ version 15.1 (StataCorp LLC, College Station, TX, USA).

\subsection{Ethics}

This study was part of the project "Near real-time automated vaccine safety signal detection using routinely collected healthcare data: Potential for continuous active surveillance" approved by the Monash Health Human Research Ethics Committee (HREC) (HREC/18/MonH/345) on 05 May 2018.

\section{Results}

Of the 249,895 ED encounters during the study period, we identified 76 ED visits with the ICD-10 diagnosis codes of T80.5, T80.6 and T88.1. Seven records (9.2\%) were excluded due to either missing records or lack of adequate information in the records (particularly progression of signs and symptoms) leading to uncertainty of the patient's diagnosis. In addition, we screened 167 records coded with T78.2 and T88.6 but did not identify any potential anaphylaxis cases due to serum/vaccination/immunisation. The remaining 69 patient records (90.8\%) were considered for further analyses (Figure 1); $57.9 \%$ were male, $53.6 \%$ were aged 0 to 10 years and $63.8 \%$ of the patients had a documented vaccination history (Table 3). The levels of agreement (Cohen's kappa score) between reviewers to categorize the identified records as anaphylaxis case or not were substantial; 0.64 for T88.1 code, 0.68 for T80.5 code, and 0.78 for T80.6 code. 


\section{Confirmed anaphylaxis diagnosis}

Based on chart review, we confirmed 29 cases of anaphylaxis; 17 (58.6\%) anaphylaxis cases were triggered by vaccination and 12 cases were associated with other causes. All cases were classified as BC case definition level $1(37.9 \%)$ or $2(62.1 \%)$, with none being categorized as level 3. Other causes triggering anaphylaxis include desensitising immunotherapy $(n=6)$, cortisone injections/antibiotics $(n=3)$ and bee sting $(n=2)$. For one case, it was unclear what triggered anaphylaxis because the patient had received vaccination and cat exposure simultaneously. The onset of symptoms among cases was within 30 minutes (13 cases), between 30 minutes and 2 hours (11 cases) and 2 to 24 hours ( 5 cases). Eighteen patients $(62.1 \%)$ presented to the ED with the chief complaint of anaphylactic reaction, six (20.7\%) with allergic reaction and the remaining five patients presented due to rash/urticaria, collapse or distress post-immunisation. Characteristics of the confirmed anaphylaxis cases are summarized in Table 3.

The overall PPV considering codes T80.5, T80.6 and T88.1 was $42 \%(29 / 69,95 \% \mathrm{Cl} 30.2$ - 54.5). However, the overall PPV could be $38.2 \%$ (29/76) when considering the number of records that were found with the code-based algorithm search as the denominator, while assuming the excluded seven records as non-anaphylaxis cases.

This article is protected by copyright. All rights reserved. 
The PPVs were highest for code T80.5 (22/23, 95.6\%) and for patients presenting to the ED with a chief complaint of anaphylactic reaction (18/19, 94.7\%). Based on age group, PPVs were higher in older children and non-elderly adults with $63.6 \%(7 / 11)$ in the $11-19$ and $73.7 \%(14 / 19)$ in the $20-64$ age category (Table 4$)$.

There were eight misclassified cases out of the 69 patients identified using the codebased algorithm: one false positive case and seven false negative cases. Out of the 23 anaphylactic cases identified using code T80.5, one case was classified as not true anaphylaxis case by human review (no major dermatological or respiratory signs and symptoms, only two major cardiovascular signs [hypotension and loss of consciousness]) of the patient medical record. Five true anaphylaxis cases, based on the $B C$ case definition, were identified out of the 26 records using code T80.6. The original diagnoses for those five patients were urticaria ( 2 cases), reaction to vaccine ( 2 cases) and febrile illness for one case. Out of the 20 records identified using code T88.1, two anaphylaxis cases were incorrectly diagnosed as seizure post-vaccination.

\section{Anaphylaxis cases triggered by vaccination/immunisation}

Of the confirmed anaphylaxis cases, 58.6\% (17/29) occurred after the patients received vaccinations. The type of vaccines administered were seasonal influenza vaccine, human papillomavirus (HPV) vaccine, diphtheria-tetanus-pertussis (DTaP) vaccine, meningococcal vaccine, pneumococcal polysaccharide vaccine (PPV23) and other vaccines routinely administered at 2, 4, 6, 12 and 18 months, and 4 years in the

This article is protected by copyright. All rights reserved. 
Australian National Immunisation program. ${ }^{15}$ Details of vaccines administered, other exposures triggered anaphylactic reaction, chief presenting complaints and primary diagnosis are presented in Supplementary File 1.

The PPVs to identify anaphylaxis following vaccination were $24.6 \%(95 \% \mathrm{Cl}: 15.1$ - 36.5$)$ and $52.2 \%(95 \% \mathrm{Cl}: 30.6-73.2)$ considering all the three codes and T80.5 alone respectively. Based upon subgroup analysis, PPV was relatively higher for the 11-19 age group (54.5\%; $95 \% \mathrm{Cl}: 23.4-83.3$ ) and for patients who presented to the ED with convulsion or distress or altered consciousness (80.0\%; 95\%Cl: $28.4-99.5)$. More than two-thirds (70.6\%) of ED visits due to anaphylaxis following vaccinations presented with chief presenting problems of either anaphylactic reaction/shock or allergic reaction. There was a substantial increment in the number of confirmed anaphylaxis following vaccination in 2017 ( $p$-value $<0.0001$ ) (Figure 2). The PPV of individual ICD10-CM codes and based on subgroup analysis are presented in Table 4.

\section{Discussion}

Our medical chart review found that ICD-10-CM code T80.5 was most predictive for anaphylaxis due to vaccination or other serum products $(95.7 \% 95 \% \mathrm{Cl}$ : $78.1-99.9)$, and was higher than estimates reported in prior studies. ${ }^{13,16}$ However, T80.5 resulted in a modest PPV to detect anaphylaxis only due to vaccination/immunisation $(52.2 \%$ (95\% Cl: $30.6-73.2)$. An additional seven cases of anaphylaxis were identified by reviewing 46 medical records identified using nonspecific codes (T80.6 and T88.1), but

This article is protected by copyright. All rights reserved. 
the PPVs considering all the three codes were below average: $43.9 \%$ for anaphylaxis due to vaccination or other serum products, and $25.8 \%$ for anaphylaxis only due to vaccination. The absence of a diagnostic code specific to anaphylaxis due to vaccination may have affected the result of this study. Moreover, under-diagnosis of anaphylaxis due to vaccination and incomplete recording of patient's vaccination history may also affect the PPV of the algorithm because of misclassification of codes.

We did not identify any previous studies that evaluated the accuracy of ICD codes to identify anaphylaxis due to vaccination. However, some have reported on the accuracy of anaphylaxis specific or nonspecific ICD codes in predicting episodes of true anaphylaxis. A study evaluated the accuracy of ICD-9 codes to identify anaphylaxis associated with medication and biologics of interest reported a PPV of $69.0 \%(95 \% \mathrm{Cl}$ : $58-78.7^{13}$. This study validated ICD-9 codes of 99.5 (other anaphylactic shocks) and 999.4 (anaphylactic shock due to serum) using ED and inpatient medical records. A higher PPV (88.4\%) of predicting anaphylaxis case was reported by another study using anaphylaxis specific ICD-10 codes in the ED setting: T78.1 (anaphylactic reaction due to food), T78.1 (anaphylactic reaction due to peanut) and T78.2 (anaphylactic reaction due shellfish). ${ }^{16}$ Both of the aforementioned studies used different gold standards to confirm anaphylaxis case: the clinical criteria for diagnosing anaphylaxis developed during the second symposium on the definition and management of anaphylaxis ${ }^{17}$ and

This article is protected by copyright. All rights reserved. 
the World Allergy Organisation (WAO) anaphylaxis clinical diagnosis criteria respectively.

We did not evaluate the sensitivity and specificity of the algorithm but we evaluated patients with codes suggestive of anaphylaxis. The difference in PPVs by principal presenting problems may be an important key to improve the accuracy of the algorithm through combination of chief presenting complaints and the ICD-10-CM codes. Over two-thirds (70.6\%) of confirmed anaphylaxis after vaccination patients presented to the ED with chief complaints of anaphylactic reaction, allergic reaction or distress/altered consciousness. Given that anaphylaxis after vaccination is underdiagnosed/missed-diagnosed and a very rare event, adding non-anaphylactic codes such as T80.6 and T88.1 may have utility in vaccine safety syndromic surveillance. In this study, patients verified as post-vaccination anaphylaxis but originally coded as non-anaphylaxis cases were coded under T88.1 (10.5\%) and T80.6 (11.5\%). Our findings demonstrate that considering anaphylaxis-specific secondary diagnosis code or associated diagnosis codes other than anaphylaxis can improve the identification of true anaphylaxis cases. ${ }^{18}$ Typically, electronic health record surveillance involves a trade-off between sensitivity and PPV, with no set "acceptable PPV" threshold defined, and likely to vary depending upon the condition surveyed. ${ }^{19}$ Given the rarity and severity of anaphylaxis post vaccination, a lower PPV may be acceptable.

This article is protected by copyright. All rights reserved. 
We observed an increase in anaphylaxis following vaccination presentations to the ED from 2010 to 2017 . Nearly half (47\%) of all the anaphylaxis presentations were seen in 2017 and 2018. While we used data from a single health network, a state-wide report from the Safer Care Victoria anaphylaxis clinical care standard in 2019 also showed that anaphylaxis presentations to Victorian public hospital emergency departments, from $2012 / 13-2016 / 17$, grew by $30 \% .^{20}$

\section{Limitations}

The generalisability of our findings to broader syndromic surveillance of anaphylaxis following vaccination over time is limited by its small sample size, with the included population from a single hospital network, albeit the largest in Victoria incorporating three separate emergency departments. We only considered medical records from the emergency department setting, and medical records were identified solely using primary diagnosis code. Discharge ICD codes were not considered in this study as they are not available in real-time in our health system and cannot yet inform potential near-real-time surveillance. The use of a variety of ICD codes have been assessed for their accuracy in vaccine safety research across institutions and health-care level (inpatient, ED, outpatient). Significant variation in accuracy was found between different codes and also between health-care levels. ${ }^{21}$ While inpatient coding related health-care funding may introduce bias in inpatient coding, ED coding is not affected by potential funding bias in our setting. This study assessed PPVs and could not

This article is protected by copyright. All rights reserved. 
evaluate sensitivity and specificity. Medical record reviewers were not blinded to the study objective. Seven of the 76 ED presentations (9.2\%) examined had missing records or insufficient clinical information to assign a diagnosis or $\mathrm{BC}$ level, limiting full assessment of coding accuracy.

\section{Conclusion}

The overall PPV of the algorithm to identify anaphylaxis due to vaccination/immunisation in the ED dataset was low. However, the PPV was improved using ICD-10-CM code of T80.5 alone. Given that there is no specific ICD-10-CM diagnosis code for anaphylaxis due to vaccination, ICD-10-CM code T80.5 may be suitable to monitor anaphylaxis due to vaccination in the ED setting. Validation of the utility of monitoring T80.5 in vaccine safety signal detection should to be confirmed in future multicentre studies.

\section{Availability of data and material}

The datasets generated and/or analysed during the current study are not publicly available as they contain sensitive information, but aggregated data are available from the corresponding author on reasonable request.

\section{Author contribution}

Yonatan M. Mesfin made substantial contribution to the conception/design of the manuscript, data analyses and drafting the paper with guidance from Jim Buttery and

This article is protected by copyright. All rights reserved. 
Allen C Cheng. Aimy HL Tran made substantial contribution to the medical records review and data analyses. All authors critically revised the paper and approved the final manuscript.

Funding: This research did not receive specific grant from funding agencies in the public, commercial or not-for-profit sectors.

\section{Disclosure}

The authors report no conflicts of interest in this work.

ORCID

Yonatan Moges Mesfin https://orcid.org/0000-0002-0010-9845

Aimy HL Tran https://orcid.org/0000-0002-7240-3867

\section{References}

1. O'Hagan DT, Rappuoli R. The safety of vaccines. Drug discovery today. 2004;9(19):84654.

2. McPhillips H, Marcuse EK. Vaccine safety. Current problems in Pediatrics. 2001;31(4):95-121.

3. Griffin MR, Braun MM, Bart KJ. What should an ideal vaccine postlicensure safety system be? American journal of public health. 2009;99(S2):S345-S50.

4. Dey A, Wang $\mathrm{H}$, Quinn $\mathrm{HE}$, et al. Surveillance of adverse events following immunisation in Australia annual report, 2014. Communicable Diseases Intelligence Quarterly Report. 2016;40(3):E377-E90.

5. Mesfin YM, Cheng A, Lawrie J, et al. Use of routinely collected electronic healthcare data for postlicensure vaccine safety signal detection: a systematic review. BMJ Global Health. 2019;4(4):e001065.

6. Mesfin YM, Cheng AC, Jock Lawrie, et al. Use of routinely collected electronic health care data for post-licensure vaccine safety signal detection: a systematic review Unpublished. 2019.

This article is protected by copyright. All rights reserved. 
7. Dey A, Gidding H, Menzies R, et al. General practice encounters following seasonal influenza vaccination as a proxy measure of early-onset adverse events. Vaccine. 2014;32(19):2204-8.

8. Nainani V, Galal U, Buttery J, et al. An increase in accident and emergency presentations for adverse events following immunisation after introduction of the group $B$ meningococcal vaccine: an observational study. Archives of disease in childhood. 2017;102(10):958-62.

9. World Health Organization. ICD-10: International statistical classification of diseases and related health problems: World Health Organization. 2004.

10. Bohlke K, Davis RL, Marcy S, et al. Risk of anaphylaxis after vaccination of children and adolescents. Pediatrics. 2003;112(4):815-20.

11. Rüggeberg JU, Gold MS, Bayas J-M, et al. Anaphylaxis: case definition and guidelines for data collection, analysis, and presentation of immunization safety data. Vaccine. 2007;25(31):5675-84.

12. McNeil MM, Weintraub ES, Duffy J, et al. Risk of anaphylaxis after vaccination in children and adults. Journal of Allergy and Clinical Immunology. 2016;137(3):868-78.

13. Walsh KE, Cutrona SL, Foy S, et al. Validation of anaphylaxis in the Food and Drug Administration's Mini-Sentinel. Pharmacoepidemiology and drug safety. 2013;22(11):1205-13.

14. Monash Health Innovate Reconciliation Action Plan (2016-2018). Available at https://monashhealth.org/wp-content/uploads/2018/06/188711 1496277909.pdf. [Internet]. Monash Health [cited 11 July 2019].

15. National Immunisation Program Schedule (Australia). Availble at https://beta.health.gov.au/health-topics/immunisation/immunisation-throughout-

life/national-immunisation-program-schedule. [Internet]. [cited 11 July 2019].

16. Uthairat M, Sakulchit T, Sangsupawanich P. Accuracy of ICD-10 Coding for Anaphylaxis. Journal of Allergy and Clinical Immunology. 2016;137(2):AB54.

17. Sampson HA, Muñoz-Furlong A, Campbell RL, et al. Second symposium on the definition and management of anaphylaxis: summary report-Second National Institute of Allergy and Infectious Disease/Food Allergy and Anaphylaxis Network symposium. Journal of Allergy and Clinical Immunology. 2006;117(2):391-7.

18. Harduar-Morano L, Simon MR, Watkins S, et al. Algorithm for the diagnosis of anaphylaxis and its validation using population-based data on emergency department visits for anaphylaxis in Florida. Journal of Allergy and Clinical Immunology. 2010;126(1):98-104. e4.

19. Birkhead GS, Klompas M, Shah NR. Uses of electronic health records for public health surveillance to advance public health. Annual review of public health. 2015;36:345-59.

20. Safer Care Victoria. Anaphylaxis clinical care standard. Safer Care Victoria (Australia), February 2019.

21. Mullooly J, Donahue J, DeStefano F, et al. Predictive value of ICD-9-CM codes used in vaccine safety research. Methods of information in medicine. 2008;47(04):328-35.

List of tables

Table 1. Brighton Collaboration case definition of anaphylaxis 
For all levels of diagnostic certainty:

Anaphylaxis is a clinical syndrome characterized by

- sudden onset AND

- rapid progression of signs and symptoms AND

- multiple ( $\geq 2$ ) organ systems

Level 1 of diagnostic certainty

$-\geq 1$ major dermatological AND

- $\geq 1$ major cardiovascular AND/OR $\geq 1$ major respiratory criterion

Level 2 of diagnostic certainty

- $\geq 1$ major cardiovascular AND $\geq 1$ major respiratory criterion

OR

- $\geq 1$ major cardiovascular OR respiratory criterion AND

- $\geq 1$ minor criterion involving $\geq 1$ different system (other than cardiovascular or respiratory systems)

- ( $\geq 1$ major dermatologic) AND ( $\geq 1$ minor cardiovascular AND/OR minor respiratory criterion

Level 3 of diagnostic certainty

- $\geq 1$ minor cardiovascular OR respiratory criterion AND

- $\geq 1$ minor criterion from each of $\geq 2$ different systems/categories

This article is protected by copyright. All rights reserved. 
Tbale 2. Major and minor criteria used in the case definition of anaphylaxis: Brighton Collaboration criteria

\begin{tabular}{|c|c|c|}
\hline Syndrome & Major criteria & Minor criteria \\
\hline $\begin{array}{l}\text { Dermatological } \\
\text { or mucosal }\end{array}$ & $\begin{array}{l}\text { - } \text { Generalized urticaria (hives) or generalized } \\
\text { erythema } \\
\text { - } \quad \text { Angioedema, } \underset{*}{*} \text { localized or generalized } \\
\text { - } \quad \text { Generalized pruritus with skin rash }\end{array}$ & $\begin{array}{l}\text { - Generalized pruritus without skin } \\
\text { rash } \\
\text { - } \text { Generalized prickle sensation } \\
\text { - Localized injection site urticarial }\end{array}$ \\
\hline $\begin{array}{l}\text { Cardiovascular } \\
\text { (major) }\end{array}$ & $\begin{array}{l}\text { - Measured hypotension } \\
\text { - Clinical diagnosis of uncompensated shock, } \\
\text { indicated by the combination of at least } 3 \text { of } \\
\text { the following: } \\
\text { - Tachycardia } \\
\text { - Capillary refill time }>3 \mathrm{~s} \\
\text { - } \text { Reduced central pulse volume } \\
\text { - Decreased level of consciousness or loss of } \\
\text { consciousness }\end{array}$ & $\begin{array}{l}\text { - Red and itchy eyes } \\
\text { - Reduced peripheral circulation as } \\
\text { indicated by the combination of at } \\
\text { least } 2 \text { of } \\
\text { - Tachycardia and } \\
\text { - A capillary refill time }>3 \mathrm{~s} \\
\text { without hypotension } \\
\text { - A decreased level of } \\
\text { consciousness }\end{array}$ \\
\hline
\end{tabular}




\begin{tabular}{|c|c|c|}
\hline Respiratory & $\begin{array}{l}\text { - Bilateral wheeze (bronchospasm) } \\
\text { - Stridor } \\
\text { - Upper airway swelling (lip, tongue, throat, } \\
\text { - Respiratory or larynx) } \\
\text { following: } \\
\text { - Tachypnoea } \\
\text { - Increased use of accessory respiratory } \\
\text { muscles (sternocleidomastoid, intercostal } \\
\text { etc) } \\
\text { - Recession } \\
\text { - Cyanosis } \\
\text { - Grunting }\end{array}$ & $\begin{array}{l}\text { - Persistent dry cough } \\
\text { - Hoarse voice } \\
\text { - Difficulty breathing without } \\
\text { wheeze or stridor } \\
\text { - Sensation of throat closure } \\
\text { - Sneezing, rhinorrhea }\end{array}$ \\
\hline Gastrointestinal & & $\begin{array}{l}\text { - Diarrhoea } \\
\text { - Abdominal pain } \\
\text { - Nausea } \\
\text { - Vomiting }\end{array}$ \\
\hline
\end{tabular}

*Not hereditary angioedema

Table 3. Demographic and diagnostic characteristics of the 69 patients identified for review in the ED dataset, 2010-2018.

\begin{tabular}{|l|c|c|c|c|}
\hline Characteristic & Number of & \multicolumn{3}{|c|}{ Anaphylaxis case level of certainty } \\
\cline { 4 - 5 } & charts & Level 1, & Level 2, & Not anaphylaxis \\
& reviewed & $n(\%)$ & $n(\%)$ & case, $\mathrm{n}(\%)$ \\
\hline Total & 69 & $11(15.9)$ & $18(26.1)$ & $40(58.0)$ \\
\hline Gender & & & & \\
\hline Female & 29 & $7(24.1)$ & $6(20.7)$ & $16(55.2)$ \\
\hline
\end{tabular}

This article is protected by copyright. All rights reserved. 


\begin{tabular}{|c|c|c|c|c|}
\hline Male & 40 & $4(10.0)$ & $12(30.0)$ & $24(60.0)$ \\
\hline \multicolumn{5}{|l|}{ Age group (years) } \\
\hline 0 to 10 & 37 & $4(10.8)$ & $3(8.1)$ & $30(81.0)$ \\
\hline 11 to 19 & 11 & $2(18.2)$ & $5(45.5)$ & $4(36.3)$ \\
\hline 20 to 64 & 19 & $5(26.3)$ & $9(47.4)$ & $5(26.3)$ \\
\hline$\geq 65$ & 2 & 0 & $1(50)$ & $1(50)$ \\
\hline \multicolumn{5}{|l|}{ ICD-10-CM codes } \\
\hline T80.5 & 23 & $7(30.4)$ & $15(65.2)$ & $1(4.4)$ \\
\hline T80.6 & 26 & $3(11.5)$ & $2(7.7)$ & $21(80.8)$ \\
\hline T88.1 & 20 & $1(5.0)$ & $1(5.0)$ & 18(90.0) \\
\hline \multicolumn{5}{|l|}{ Vaccination received } \\
\hline Yes & 44 & $7(15.9)$ & $11(25.0)$ & $26(59.1)$ \\
\hline No/Not documented & 25 & $4(16.0)$ & $7(28.0)$ & $14(56.0)$ \\
\hline \multicolumn{5}{|c|}{ Chief presenting complaints } \\
\hline Anaphylactic reaction & 19 & $6(31.6)$ & $12(63.2)$ & $1(5.3)$ \\
\hline Allergic reaction & 11 & $3(27.3)$ & $3(27.3)$ & $5(45.4)$ \\
\hline Others & 39 & $2(5.1)$ & $3(7.7)$ & $34(87.2)$ \\
\hline
\end{tabular}

Table 4. Positive predictive value for each ICD-10 code, study period and subgroup of patients.

\begin{tabular}{|l|l|l|l|l|l|}
\hline $\begin{array}{l}\text { Characteri } \\
\text { stics }\end{array}$ & $\begin{array}{l}\text { Number } \\
\text { of charts } \\
\text { reviewed }\end{array}$ & $\begin{array}{l}\text { Anaphylaxis } \\
\text { Confirmed } \\
\text { regardless of }\end{array}$ & PPV $(95 \% \mathrm{Cl})$ & $\begin{array}{l}\text { Anaphylaxi } \\
\text { s after } \\
\text { vaccination }\end{array}$ & PPV $(95 \% \mathrm{Cl})$ \\
\hline
\end{tabular}




\begin{tabular}{|c|c|c|c|c|c|}
\hline & (n) & cause (n) & & (n) & \\
\hline \multicolumn{6}{|c|}{ ICD 10 codes } \\
\hline T80.5 & 23 & 22 & $95.6(78.1-99.9)$ & 12 & $52.2(30.6-73.2)$ \\
\hline T80.6 & 26 & 5 & $19.2(6.6-39.4)$ & 3 & $11.5(2.4-30.2)$ \\
\hline T88.1 & 20 & 2 & $10(1.2-31.7)$ & 2 & $10.0(1.2-31.6)$ \\
\hline All codes & 69 & 29 & $42(34.2-54.5)$ & 17 & $24.6(15.1-36.5)$ \\
\hline \multicolumn{6}{|l|}{ Gender } \\
\hline Male & 40 & 16 & $40.0(24.9-56.7)$ & 8 & $20.0(9.1-35.6)$ \\
\hline Female & 29 & 13 & $44.8(26.4-64.3)$ & 9 & $31.0(15.3-50.8)$ \\
\hline \multicolumn{6}{|c|}{ Age groups (years) } \\
\hline $0-10$ & 37 & 7 & $18.9(7.9-35.2)$ & 5 & $13.5(4.5-28.8)$ \\
\hline $11-19$ & 11 & 7 & $63.6(30.8--89.1)$ & 6 & $54.5(23.4-83.3)$ \\
\hline $20-64$ & 19 & 14 & 73.7 (48.8 - 90.9) & 6 & $31.6(12.6-56.6)$ \\
\hline $\begin{array}{l}65 \text { and } \\
\text { older }\end{array}$ & 2 & 1 & $50.0(12.6-56.7)$ & 0 & - \\
\hline \multicolumn{6}{|c|}{ Chief presenting complaints } \\
\hline $\begin{array}{l}\text { Anaphylac } \\
\text { tic } \\
\text { reaction }\end{array}$ & 19 & 18 & 94.7 (73.9 - 99.9) & 8 & $42.1(20.3-66.5)$ \\
\hline $\begin{array}{l}\text { Allergic } \\
\text { reaction }\end{array}$ & 11 & 6 & $54.5(23.4-83.3)$ & 4 & $34.4(10.9-69.2)$ \\
\hline $\begin{array}{l}\text { Convulsio } \\
\text { n/distress }\end{array}$ & 5 & 4 & $80.0(28.4-99.5)$ & 4 & $80.0(28.4-99.5)$ \\
\hline Others & 34 & 1 & $2.9(0.7-15.3)$ & 1 & $2.9(0.7-15.3)$ \\
\hline \multicolumn{6}{|c|}{ Study period } \\
\hline $2010-2014$ & 28 & 9 & $32.1(15.9-52.4)$ & 7 & $25.0(10.7-44.9)$ \\
\hline
\end{tabular}




\begin{tabular}{|l|l|l|l|l|l|}
\hline $2015-2018$ & 41 & 20 & $48.8(32.9-64.9)$ & 10 & $24.4(12.4-40.3)$ \\
\hline
\end{tabular}

T80.5 - anaphylactic reaction due to serum/ vaccine; T88.1 - other complication following immunisation not elsewhere classified; T80.6 - other serum reaction, not anaphylaxis

This article is protected by copyright. All rights reserved. 


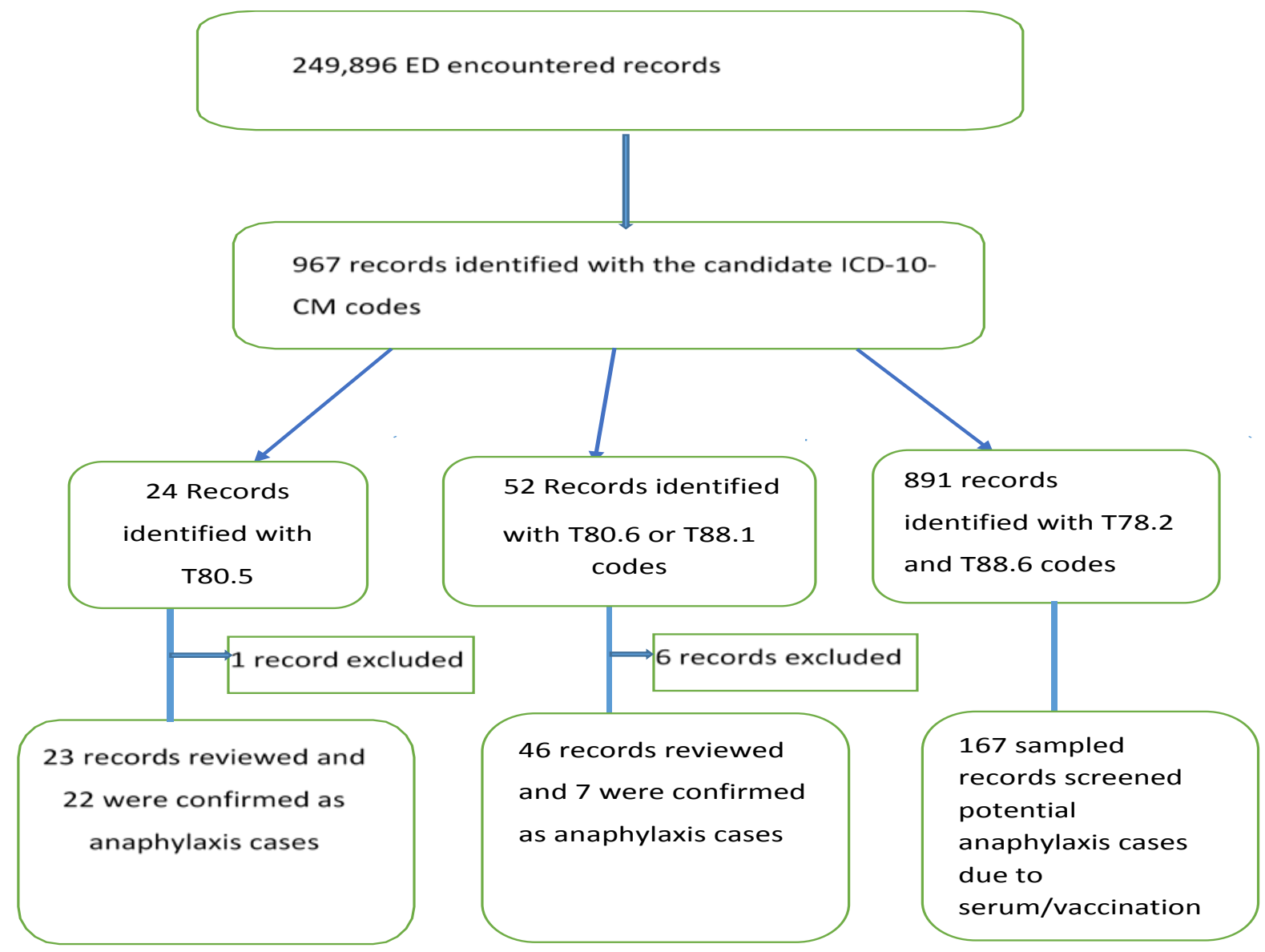

Figure1. ICD-10-CM coding algorithm to identify emergency department visits for anaphylaxis due to vaccination. Records were excluded due to either missing records or lack of adequate information in the records. 
12

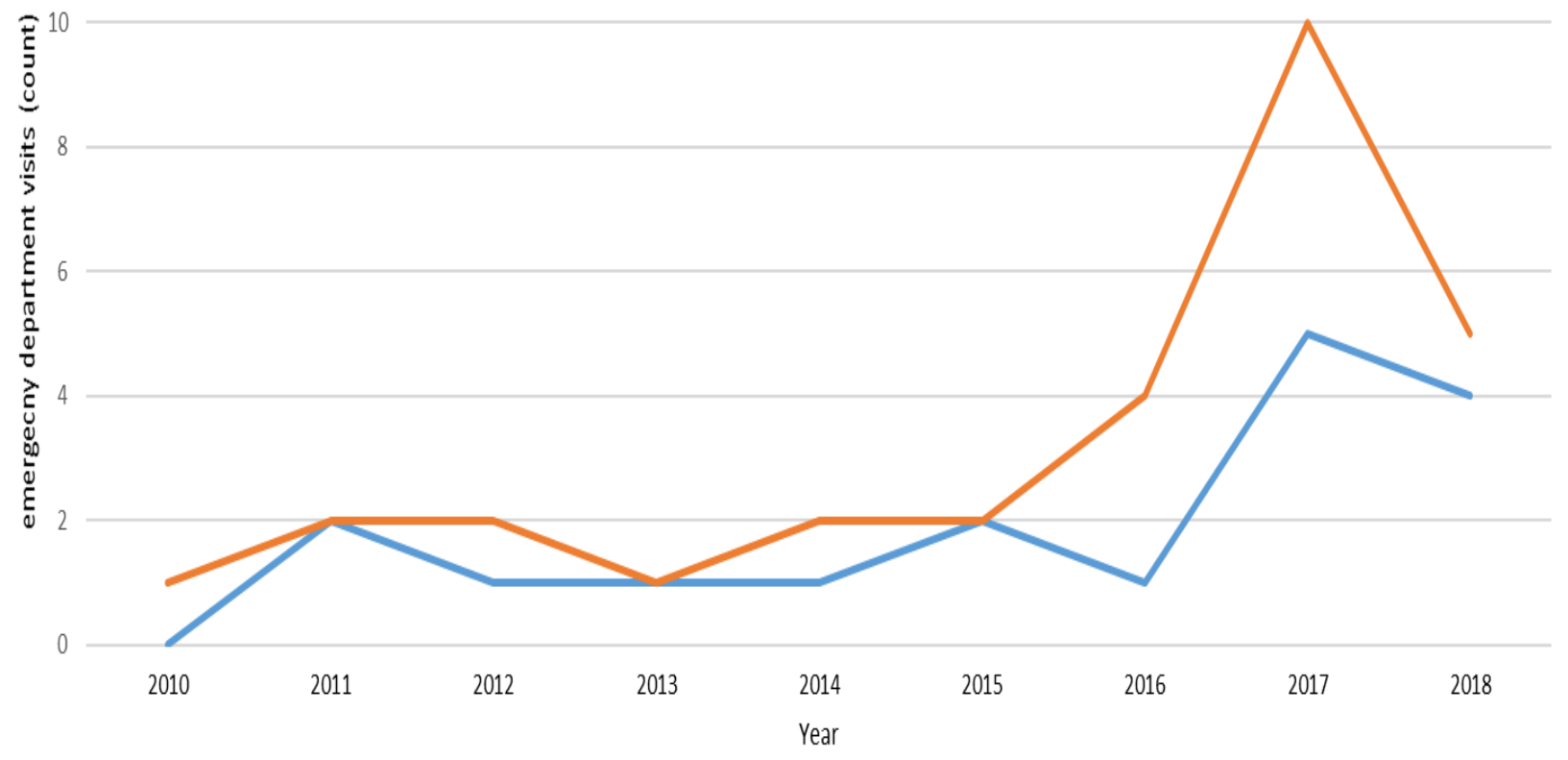

-Anaphylaxis due to vaccine/immunisation -Anaphylaxis due to serum/vaccine/immunisations

Figure 2 Figure 2. Annual number of anaphylaxis presentations to the Monash Health emergency department between 2010 and 2018.

This article is protected by copyright. All rights reserved. 


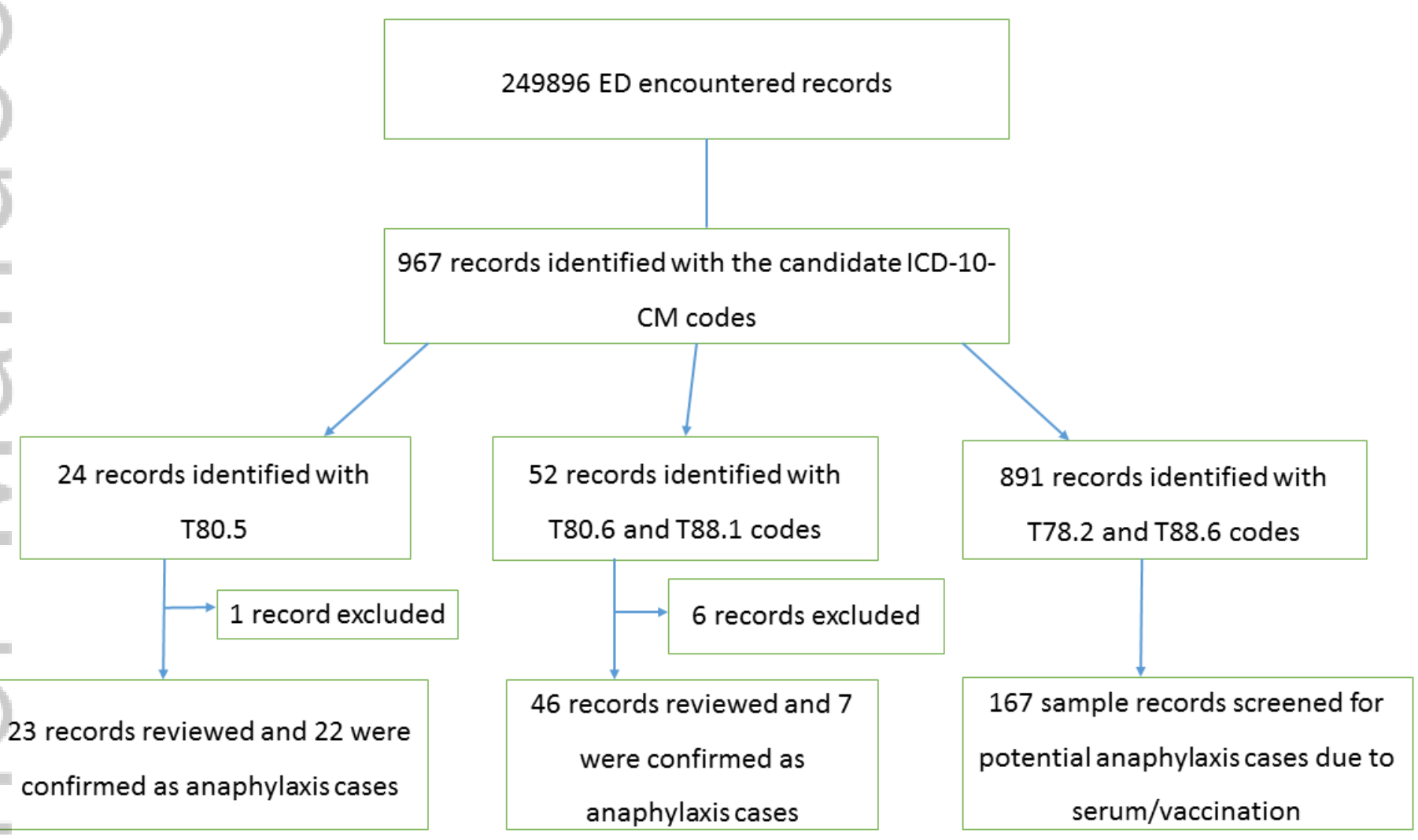

This article is protected by copyright. All rights reserved. 


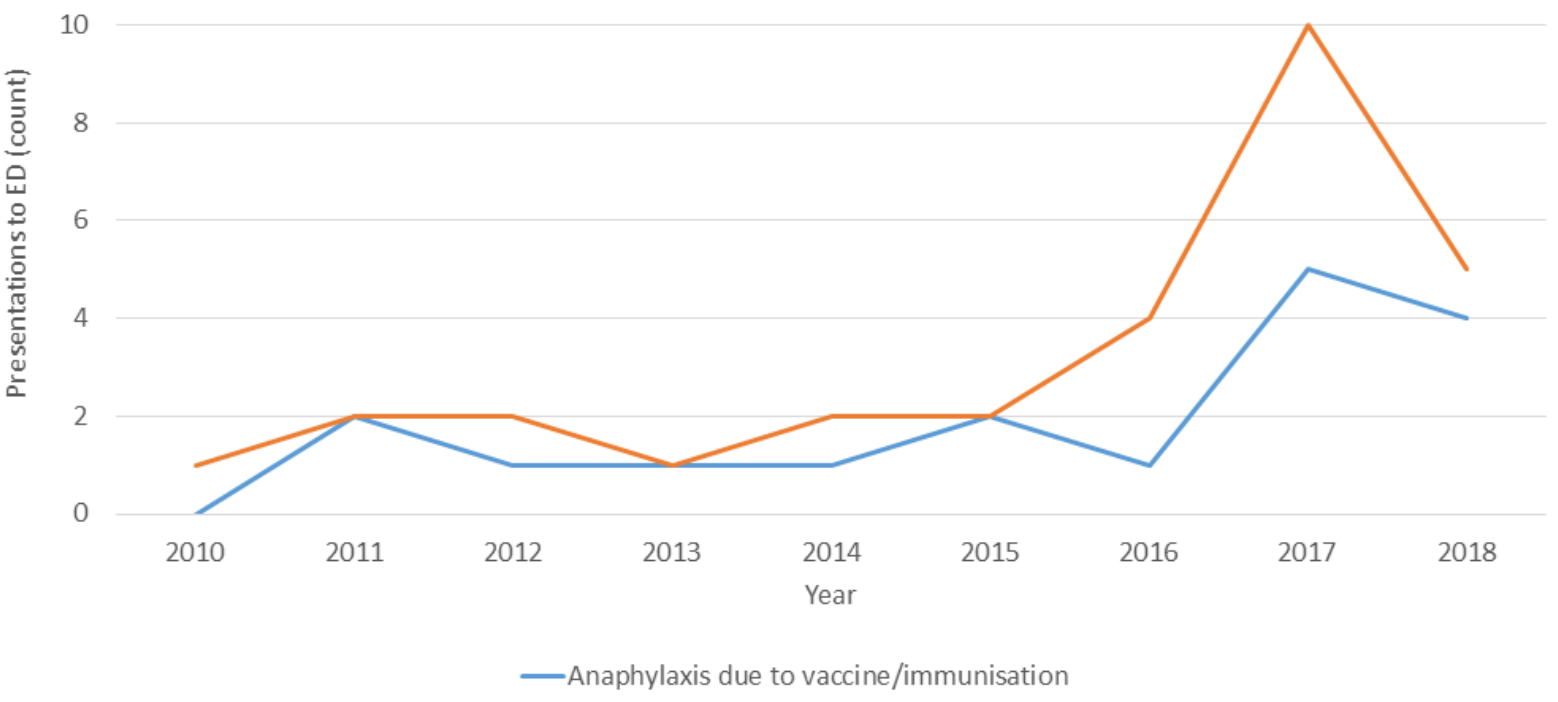

This article is protected by copyright. All rights reserved. 


\section{University Library}

\section{- M M N E R VA A gateway to Melbourne's research publications}

Minerva Access is the Institutional Repository of The University of Melbourne

Author/s:

Mesfin, YM;Cheng, AC;Tran, AHL;Buttery, J

Title:

Positive predictive value of ICD-10 codes to detect anaphylaxis due to vaccination: A validation study.

Date:

2019-10

Citation:

Mesfin, Y. M., Cheng, A. C., Tran, A. H. L. \& Buttery, J. (2019). Positive predictive value of ICD-10 codes to detect anaphylaxis due to vaccination: A validation study..

Pharmacoepidemiol Drug Saf, 28 (10), pp.1353-1360. https://doi.org/10.1002/pds.4877.

Persistent Link:

http://hdl.handle.net/11343/286326 